\title{
Methods in Molecular Biology
}

Series Editor

John M. Walker

School of Life Sciences

University of Hertfordshire

Hatfield, Hertfordshire, AL10 9AB, UK

For further volumes:

http://www.springer.com/series/7651 



\title{
Neuropeptides
}

\section{Methods and Protocols}

\author{
Edited by \\ Adalberto Merighi
}

Dipartimento di Morfofisiologia Veterinaria, Università degli Studi di Torino, Grugliasco, TO, Italy; Istituto Nazionale di Neuroscienze (INN), Università degli Studi di Torino, Grugliasco, TO, Italy 


\section{Editor}

Adalberto Merighi

Dipartimento di Morfofisiologia Veterinaria

Università degli Studi di Torino

and

Istituto Nazionale di Neuroscienze (INN)

Università degli Studi di Torino

Grugliasco, TO, Italy

adalberto.merighi@unito.it

Please note that additional material for this book can be downloaded from

http://extras.springer.com

ISSN 1064-3745

ISBN 978-1-61779-309-7

DOI 10.1007/978-1-61779-310-3

Springer New York Dordrecht Heidelberg London
e-ISSN 1940-6029

e-ISBN 978-1-61779-310-3

Library of Congress Control Number: 2011936011

(C) Springer Science+Business Media, LLC 2011

All rights reserved. This work may not be translated or copied in whole or in part without the written permission of the publisher (Humana Press, c/o Springer Science+Business Media, LLC, 233 Spring Street, New York, NY 10013, USA), except for brief excerpts in connection with reviews or scholarly analysis. Use in connection with any form of information storage and retrieval, electronic adaptation, computer software, or by similar or dissimilar methodology now known or hereafter developed is forbidden.

The use in this publication of trade names, trademarks, service marks, and similar terms, even if they are not identified as such, is not to be taken as an expression of opinion as to whether or not they are subject to proprietary rights.

Printed on acid-free paper

Humana Press is part of Springer Science+Business Media (www.springer.com) 


\section{Preface}

The term neuropeptide was originally coined to indicate small protein molecules that are contained in neurons. In the late 1970s and the 1980s of the last century, several tens of neuropeptides were localized by immunocytochemistry to discrete cell populations of the central and peripheral nervous system, and the concept of chemical neuroanatomy, originally developed by Tomas Hökfelt and coworkers, entered the scene of neurobiology. Since then, the field of neuropeptide biology has dramatically widened, and today the ultimate frontiers in neuropeptide research lie in the development of pharmacologically active compounds that are capable of crossing the blood-brain barrier to exert their biological role(s) in vivo and in the construction of genetic vectors to be employed in gene therapy.

This book represents a readily reproducible collection of established and emerging techniques for neuropeptide research. Such a collection is preceded by a general introductory chapter (Chapter 1 ) that discusses a series of new concepts leading to a broader neuropeptide definition in light of the huge amount of data accumulated after more than half a century of neuropeptide research.

The methods presented include immunocytochemical localization, biochemical characterization, functional analysis, development and production of genetic probes, and the design of neuropeptide derivatives for cellular neurobiology as well as the potential therapeutic applications.

As a general indication to the readers, Chapters 2-10 are focused on a series of techniques for localization studies. They cover a broad range of protocols, such as the immunocytochemical detection of neuropeptides in nonmammalian vertebrates together with a detailed description of procedures for anesthesia and tissue preparations in these species (Chapter 2); the combined neuropeptide/receptor localization at the light and transmission electron microscope for connectivity studies (Chapter 3); the analysis of neuropeptide genes' transcription by localization of pre-mRNA (Chapter 6) or mRNA/microRNA with in situ hybridization (Chapter 4), in situ PCR (Chapter 5), and laser capture/microdissection (Chapter 7); the visualization in vivo of neuropeptide secretion (Chapter 8) and translocation across the plasma membrane (Chapter 9); and the functional analysis of neuropeptide interactions in vitro with cells of the immune system (Chapter 10).

Chapter 11 describes a series of electrophysiological protocols for functional studies in vitro and in vivo.

Chapters 12-19 are devoted to biochemical/molecular biology techniques, ranging from radioimmunoassay (Chapter 12) to neuropeptidomics employing reverse-phase HPLC (Chapter 13) or mass spectrometry (Chapter 14), RNA analysis by suppression subtractive hybridization (Chapter 15), determination of neuropeptide release in vivo by microdialysis (Chapter 16) or antibody microprobes (Chapter 17), and measurement of neuropeptidases (Chapter 18) and neuropeptide autoantibody levels (Chapter 19) in biological fluids.

Chapters 20-24 deal with a number of techniques developed to optimize neuropeptide administration to central neurons or to interfere with biological effects in vivo. These procedures include the intranasal delivery of neuropeptides (Chapter 20), the development of 
neuropeptide pro-drugs (Chapter 21), the use of phosphorothioate oligodeoxynucleotides that are capable of crossing the blood-brain barrier to knock down neuropeptides in the CNS (Chapter 22), the development of liposome-encapsulated neuropeptides for assessing the chronic actions of physiologically short-lived molecules (Chapter 23), the construction of recombinant adeno-associated viral vectors that can be used to locally or systemically enhance or silence neuropeptide gene expression (Chapter 24).

Finally, Chapter 25 describes a calcium mobilization assay in mammalian cells to identify novel G-protein-coupled receptor family members that transduce the neuropeptide signals.

All scientists who have excellently contributed to this book have a direct experience in one or more fields of neuropeptide research. I am very much indebted to all of them for their successful effort in emphasizing the description of the more common pitfalls in the techniques that they have described and of the hints to reduce the possibility of failure for beginners.

The collection of protocols that forms this book is surely not exhaustive of the wide range of approaches that today can be employed in top level neuropeptide research. Yet it is intended for a large audience of scientists, including histologists, biochemists, cellular and molecular biologists, and electrophysiologists that are currently active in the field or are willing to enter such an exciting and still expanding area of neurobiology. 


\section{Contents}

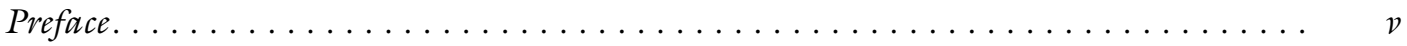

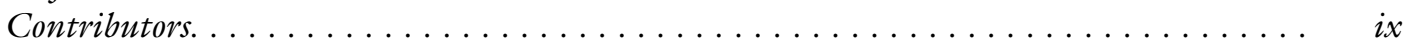

1 What Are Neuropeptides? .............................. 1 J. Peter H. Burbach

2 Neuropeptide Localization in Nonmammalian Vertebrates . . . . . . . . . . . . . . 37 Paolo de Givolamo and Carla Lucini

3 Combined Light and Electron Microscopic Visualization of Neuropeptides and Their Receptors in Central Neurons . . . . . . . . . . . . . . . . . . 57 Chiara Salio, Laura Lossi, and Adalberto Merigbi

4 Neuropeptide RNA Localization in Tissue Sections. . . . . . . . . . . . . . . . . . 73 Marc Landry, Shérine Abdel Salam, and Marie Moftah

5 Intron-Specific Neuropeptide Probes . . . . . . . . . . . . . . . . . . . . . . . 89 Havold Gainer, Todd A. Ponzio, Chunmei Yue, and Makoto Kawasaki

6 Direct In Situ RT-PCR . . . . . . . . . . . . . . . . . . . . . . 111 Laura Lossi, Graziana Gambino, Chiara Salio, and Adalberto Merigbi

7 Laser Capture Microdissection and Quantitative-PCR Analysis . . . . . . . . . . . 127 Sarab J. Paulsen and Leif K. Larsen

8 Visualization of Peptide Secretory Vesicles in Living Nerve Cells............ . 137 Joshua J. Park and $\Upsilon$. Peng Loh

9 Fluorescence Imaging with Single-Molecule Sensitivity and Fluorescence Correlation Spectroscopy of Cell-Penetrating Neuropeptides . . . . . . . . . . . . . 147 Vladana Vukojević, Astrid Gröslund, and Georgy Bakalkin

10 Analysis of Neuroimmune Interactions by an In Vitro Coculture Approach. . . . . 171 Tadabide Furuno and Mamoru Nakanishi

11 Electrophysiology ................................ 181 Zhi-Qing David Xu

12 Localization of Neuropeptides by Radioimmunoassay .................. 191 Fred Nyberg and Mathias Hallberg

13 Reversed-Phase HPLC and Hyphenated Analytical Strategies for Peptidomics . . . . . . . . . . . . . . . . . . . . . . . . . . . . . 203 Anne-Marie Hesse, Sega Ndiaye, and Joelle Vinh

14 Neuropeptidomics: Mass Spectrometry-Based Qualitative and Quantitative Analysis ............................ 223 Ping Yin, Xiaowen Hou, Elena V. Romanova, and Jonathan V. Sweedler

15 Suppression Subtractive Hybridization . . . . . . . . . . . . . . . . . . . 237 Mobamed T. Ghorbel and David Murphy 
16 Neuropeptide Microdialysis in Free-Moving Animals . . . . . . . . . . . . . 261 Tetsuya Kushikata and Kazuyoshi Hirota

17 Antibody Microprobes for Detecting Neuropeptide Release

Rebecca J. Steagall, Carole A. Williams, and Arthur W. Duggan

18 Neuropeptidases.

Manuel Ramirez, Isabel Prieto, Inmaculada Banegas, Ana B. Segarra, and Francisco Alba

19 Neuropeptide Autoantibodies Assay Sergueï O. Fetissov

20 Intranasal Delivery of Neuropeptides Michael C. Veronesi, Daniel J. Kubek, and Michael J. Kubek

21 Prodrug Design for Brain Delivery of Small- and Medium-Sized Neuropeptides

Katalin Prokai-Tatrai and Laszlo Prokai

22 Measurement of Phosphorothioate Oligodeoxynucleotide Antisense

Transport Across the Blood-Brain Barrier. . . . . . . . . . . . . . . . . . . .

William A. Banks

23 Liposome-Encapsulated Neuropeptides for Site-Specific Microinjection

Frédéric Frézard, Robson A.S. dos Santos, and Marco A.P. Fontes

24 Recombinant Adeno-Associated Viral Vectors .

Marijke W.A. de Backer, Keith M. Garner, Mieneke C.M. Luijendijk, and Roger A.H. Adan

25 Deorphanizing G Protein-Coupled Receptors by a Calcium Mobilization Assay . .

Isabel Beets, Marleen Lindemans, Tom Janssen, and Peter Verleyen

Index. . 


\section{Contributors}

Shérine Abdel SAlam • Department of Zoology, University of Alexandria, Alexandria, Egypt

Roger A.H. AdAN - Department of Neuroscience and Pharmacology,

Rudolf Magnus Institute of Neuroscience, Utrecht University Medical Centre Utrecht, Utrecht, The Netherlands

Francisco AlBa - Department of Biochemistry and Molecular Biology III

and Immunology, University of Granada Medical School, Granada, Spain

MARIJKe W.A. DE BACKer - Department of Neuroscience and Pharmacology,

Rudolf Magnus Institute of Neuroscience, Utrecht University Medical Centre Utrecht, Utrecht, The Netherlands

Georgy BAKALKIn - Department of Pharmacentical Biosciences, Uppsala University, Uppsala, Sweden

Inmaculada Banegas - Unit of Physiology, Department of Health Sciences,

University of Jaén, Jaén, Spain

William A. Banks - Geriatrics Research, Education, and Clinical Center,

Puget Sound Health Care System, Seattle, WA, USA; Division of Gerontology

and Geriatric Medicine, Department of Internal Medicine,

University of Washington, Seattle, WA, USA

J. Peter H. Burbach - Rudolf Magnus Institute of Neuroscience,

Department of Neuroscience and Pharmacology, University Medical Center Utrecht, Utrecht, The Netherlands

IsABel BeETs - Research Group of Functional Genomics and Proteomics, K. U. Leuven, Leuven, Belgium

Arthur W. Duggan - Department of Preclinical Sciences, Royal Dick School of Veterinary Medicine, Edinburgh University, Edinburgh, Scotland, UK

SERGueï O. FeTISsov - Digestive System and Nutrition Laboratory (ADEN EA4311), Rouen University, Rouen, France

Marco A.P. Fontes - Departamento de Fisiologia e Biofísica, Instituto de Ciências Biológicas, Universidade Federal de Minas Gerais, Belo Horizonte, Minas Gerais, Brazil

FrédérIC Frézard - Departamento de Fisiologia e Biofísica, Instituto de Ciências

Biológicas, Universidade Federal de Minas Gerais, Belo Horizonte, Minas Gerais, Brazil

TAdAhide Furuno - School of Pharmacy, Aichi Gakuin University, Nagoya, Japan Harold Gainer - Laboratory of Neurochemistry, National Institute of Neurological Disorders and Stroke, National Institutes of Health, Bethesda, MD, USA

Graziana Gambino • Dipartimento di Morfofisiologia Veterinaria,

Università degli Studi di Torino, Grugliasco, TO, Italy 
Keith M. Garner • Department of Neuroscience and Pharmacology, Rudolf Magnus Institute of Neuroscience, Utrecht University Medical Centre Utrecht, Utrecht, The Netherlands

Mohamed T. GHORBel • Bristol Heart Institute, University of Bristol, Bristol, UK

Paolo de Girolamo - Department of Biological Structures, Functions and Technology, University of Naples Federico II, Naples, Italy

Astrid GRÄSLUnd • Department of Biochemistry and Biophysics, Stockbolm University, Stockholm, Sweden

Mathias Hallberg • Division of Biological Research on Drug Dependence, Department of Pharmacentical Biosciences, Uppsala University, Uppsala, Sweden Anne-Marie Hesse - Laboratory of Biological Mass Spectrometry and Proteomics (SMBP), CNRS USR3149, ESPCI ParisTech, Paris, France

Kazuyoshi Hirota • Department of Anesthesiology, Hirosaki Graduate School of Medicine, Hirosaki, Japan

XiaOwen Hou • Center for Biophysics and Computational Biology, University of Illinois, Urbana, IL, USA; Beckman Institute, University of Illinois, Urbana, IL, USA

Tom Janssen - Research Group of Functional Genomics and Proteomics, K.U. Leuven, Leuven, Belgium

Makoto Kawasaki - Department of Orthopaedics, School of Medicine, University of Occupational and Environmental Health, Kitakyushu-City, Fukuoka, Japan

DANIEL J. KuBEK - Department of Anatomy and Cell Biology, Indiana University School of Medicine, Indianapolis, IN, USA

Michael J. KubeK • Department of Anatomy and Cell Biology and Program in Medical Neuroscience, Indiana University School of Medicine, Indianapolis, IN, USA

Tetsuya Kushikata • Department of Anesthesiology, Hirosaki University Hospital, Hirosaki, Japan

MARC LANDRY - INSERM U862, University of Bordeaux, Bordeaux, France

LeIf K. LARSEN • Molecular Biology, Vipergen, Copenhagen, Denmark

Marleen Lindemans - Research Group of Functional Genomics and Proteomics, K.U. Leuven, Leuven, Belgium

Laura Lossi - Dipartimento di Morfofisiologia Veterinaria, Università degli Studi di Torino, Grugliasco, TO, Italy; Istituto Nazionale di Neuroscienze (INN), Università degli Studi di Torino, Grugliasco, TO, Italy

CARLA Lucini - Department of Biological Structures, Functions and Technology, University of Naples Federico II, Naples, Italy

MieneKe C.M. LuijendijK • Department of Neuroscience and Pharmacology, Rudolf Magnus Institute of Neuroscience, Utrecht University Medical Centre Utrecht, Utrecht, The Netherlands

Adalberto Merighi • Dipartimento di Morfofisiologia Veterinaria, Università degli Studi di Torino, Grugliasco, TO, Italy; Istituto Nazionale di Neuroscienze (INN), Università degli Studi di Torino, Grugliasco, TO, Italy

Marie Moftah - Department of Zoology, University of Alexandria, Alexandria, Egypt

DAvid MURPHY • Henry Wellcome Laboratories for Integrative Neuroscience and Endocrinology, University of Bristol, Bristol, UK

Mamoru NaKanishi - School of Pharmacy, Aichi Gakuin University, Nagoya, Japan 
SEGA NDIAYE - Laboratory of Biological Mass Spectrometry and Proteomics (SMBP), CNRS USR 3149, ESPCI ParisTech, Paris, France

Fred Nyberg • Division of Biological Research on Drug Dependence,

Department of Pharmacentical Biosciences, Uppsala University, Uppsala, Sweden

Joshua J. Park - Neurosciences, University of Toledo College of Medicine,

Toledo, OH, USA

Sarah J. Paulsen • Gubra, Hørsholm, Denmark

Y. Peng LoH • Eunice Kennedy Shriver National Institute of Child Health and Human Development, National Institutes of Health, Bethesda, MD, USA

Todd A. Ponzio - Laboratory of Neurochemistry, National Institute of Neurological

Disorders and Stroke, National Institutes of Health, Bethesda, MD, USA

Isabel Prieto - Unit of Physiology, Department of Health Sciences, University of Jaén, Jaén, Spain

Laszlo Prokai - Department of Pharmacology and Neuroscience, University of North Texas Health Science Center, Fort Worth, TX, USA

Katalin Prokai-Tatrai - Department of Pharmacology and Neuroscience,

University of North Texas Health Science Center, Fort Worth, TX, USA

Manuel Ramírez - Unit of Physiology, Department of Health Sciences,

University of Jaén, Jaén, Spain

Elena V. Romanova - Department of Chemistry, University of Illinois, Urbana, IL, USA; Beckman Institute, University of Illinois, Urbana, IL, USA

Chiara SAlio - Dipartimento di Morfofisiologia Veterinaria, Università degli Studi di Torino, Grugliasco, TO, Italy

Robson A.S. Dos SAntos - Departamento de Fisiologia e Biofísica, Instituto de Ciências

Biológicas, Universidade Federal de Minas Gerais, Belo Horizonte, Minas Gerais, Brazil

Ana B. Segarra - Unit of Physiology, Department of Health Sciences,

University of Jaén, Jaén, Spain

Jonathan V. Sweedler - Department of Chemistry, University of Illinois, Urbana,

IL, USA; Beckman Institute, University of Illinois, Urbana, IL, USA

Rebecca J. Steagall - Department of Physiology, Quillen College of Medicine, East Tennessee State University, Johnson City, TN

Peter Verleyen - Research Group of Functional Genomics and Proteomics,

K.U. Leuven, Leuven, Belgium

Michael C. Veronesi - Program in Medical Neuroscience, Indiana University School of Medicine, Indianapolis, IN, USA

Joelle Vinh • Laboratory of Biological Mass Spectrometry and Proteomics (SMBP),

CNRS USR3149, ESPCI ParisTech, Paris, France

Vladana Vukojević • Department of Clinical Neuroscience, Karolinska Institute, Stockbolm, Sweden

Carole A. Williams ${ }^{\dagger}$ - Department of Physiology, Quillen College of Medicine, East Tennessee State University, Johnson City, TN, USA

ZHI-QING David Xu • Department of Neuroscience, Karolinska Institutet, Stockholm, Sweden

PING YIN - Department of Chemistry, University of Illinois, Urbana, IL, USA;

Beckman Institute, University of Illinois, Urbana, IL, USA

Chunmei Yue - Institute of Biochemistry and Cell Biology, Shanghai Institutes for Biological Sciences, Chinese Academy of Sciences, Shanghai, China 
